BMJ Open Diabetes

Research \& Care

\title{
Efficacy and safety of efpeglenatide in key patient subgroups from the BALANCE randomized trial, stratified by pre-diabetes status, BMI, and age at baseline
}

\author{
Richard E Pratley (D) , ${ }^{1}$ Stephan Jacob, ${ }^{2}$ Seungjae Baek, ${ }^{3}$ Michael E Trautmann, ${ }^{4}$ \\ Marcus Hompesch (i) , ${ }^{4}$ OakPil Han, ${ }^{3}$ John Stewart, ${ }^{5}$ Christopher H Sorli, ${ }^{6}$ \\ Alka Shaunik, ${ }^{6}$ Kun-Ho Yoon ${ }^{7}$
}

\begin{abstract}
To cite: Pratley RE, Jacob S, Baek S, et al. Efficacy and safety of efpeglenatide in key patient subgroups from the BALANCE randomized trial, stratified by pre-diabetes status, BMI, and age at baseline. BMJ Open Diab Res Care 2022;10:e002207. doi:10.1136/
\end{abstract}

bmjdrc-2021-002207

\begin{abstract}
- Additional supplemental material is published online only. To view, please visit the journal online (http://dx.doi. org/10.1136/bmjdrc-2021002207).
\end{abstract}

For 'Presented at statement' see end of article.

Received 16 February 2021 Accepted 7 October 2021

Check for updates

(c) Author(s) (or their employer(s)) 2022. Re-use permitted under CC BY-NC. No commercial re-use. See rights and permissions. Published by BMJ.

For numbered affiliations see end of article.

Correspondence to Dr Richard E Pratley; Richard.Pratley.MD@ AdventHealth.com

\section{ABSTRACT}

Introduction Efpeglenatide is a long-acting glucagon-like peptide-1 receptor agonist being developed to improve glycemic control in type 2 diabetes (T2D). In the BALANCE 205 study (NCT02075281), efpeglenatide significantly reduced body weight versus placebo in patients with obesity, or overweight with comorbidities, and without T2D. These subanalyses explore the efficacy and safety of efpeglenatide in subgroups of patients with pre-diabetes and stratified by body mass index (BMI) or age from the BALANCE study.

Research design and methods The 20-week BALANCE study randomized patients with $\mathrm{BMl} \geq 30 \mathrm{~kg} / \mathrm{m}^{2}$ or $\geq 27 \mathrm{~kg} /$ $\mathrm{m}^{2}$ with comorbidities, and without diabetes, to efpeglenatide $4 \mathrm{mg}$ or $6 \mathrm{mg}$ once weekly, $6 \mathrm{mg}$ or $8 \mathrm{mg}$ once every 2 weeks, or placebo. For these subanalyses, patients were stratified by pre-diabetes status (glycated hemoglobin $\left(\mathrm{HbA}_{1 \mathrm{c}}\right)$ 5.7\%-6.4\% (39-46 mmol/mol) or fasting plasma glucose (FPG) 100$125 \mathrm{mg} / \mathrm{dL})$ and by BMl or age $<$ or $\geq$ median values $(34.9 \mathrm{~kg} /$ $\mathrm{m}^{2}$ and 44 years, respectively) at baseline.

Results In patients with pre-diabetes at baseline, all efpeglenatide doses led to greater proportions of patients reverting to normoglycemia $(40.6 \%-64.3 \%)$ versus placebo (10.0\%), and greater reductions in $\mathrm{HbA}_{1 \mathrm{c}}(0.30 \%-0.38 \%), \mathrm{FPG}$ (7.7-14.1 mg/dL), and weight (5.6-7.3 kg) versus placebo (nominal $p<0.05$ for all). In patients with BMl or age $<$ or $\geq$ median, greater reductions in weight were observed with all efpeglenatide doses versus placebo (nominal $p<0.01$ for all). The most common adverse events in patients receiving efpeglenatide across patient subgroups were gastrointestinal adverse events.

Conclusions These results are consistent with the overall BALANCE population and suggest beneficial effects of efpeglenatide on glycemic control and body weight regardless of pre-diabetes status, age, or BMl at baseline. The effects of efpeglenatide on glycemic control in patients with pre-diabetes suggest it might help reduce the likelihood of at-risk patients developing diabetes.

\section{INTRODUCTION}

Worldwide, the rates of overweight and obesity are at epidemic levels and continue to rise. ${ }^{1-3}$ Excess weight is associated with an increased

\section{Significance of this study}

What is already known about this subject?

- In the BALANCE 205 study, efpeglenatide, a longacting glucagon-like peptide-1 receptor agonist in development to improve glycemic control in type 2 diabetes (T2D), significantly reduced body weight versus placebo in patients with obesity or overweight with comorbidities and without T2D.

What are the new findings?

- In this subanalysis of the BALANCE 205 study, in patients with pre-diabetes at baseline, efpeglenatide led to greater proportions of patients reverting to normoglycemia versus placebo.

- Greater reductions were also seen in glycated hemoglobin $\left(\mathrm{HbA}_{1 \mathrm{c}}\right)$, fasting plasma glucose, and weight with efpeglenatide versus placebo.

$\checkmark$ In patients with body mass index (BMI) and age < or $\geq$ the median, greater reductions in weight were observed with efpeglenatide versus placebo.

How might these results change the focus of research or clinical practice?

- Results from these subgroup analyses suggest beneficial effects of efpeglenatide on glycemic control and body weight regardless of pre-diabetes status, age, or BMI at baseline.

- The effects of efpeglenatide on glycemic control in patients with pre-diabetes suggest it might help reduce the likelihood of at-risk patients developing diabetes.

- Overall, these results suggest a potential role of efpeglenatide in the management of pre-diabetes and obesity.

risk of cardiometabolic syndrome and negative health consequences including hypertension, cardiovascular disease, type 2 diabetes (T2D), cancer, and premature mortality. Inflammation associated with increasing 
age, also termed 'inflammageing', is characterized by chronically elevated levels of proinflammatory cytokines, such as interleukin 6 and tumor necrosis factor- $\alpha$, and is thought to be one of the factors that underpin the increased risk of all of these disorders as well as others. ${ }^{45}$ Age-related risk factors for T2D also include decreased beta-cell function and increased insulin resistance. ${ }^{5-7}$

Pre-diabetes, often associated with obesity, is defined in the 2021 American Diabetes Association Standards of Care as the presence of impaired fasting glucose and/ or impaired glucose tolerance and/or glycated hemoglobin $\left(\mathrm{HbA}_{1 \mathrm{c}}\right) 5.7 \%-6.4 \%(39-47 \mathrm{mmol} / \mathrm{mol}) .{ }^{8}$ Similar to obesity, meeting these criteria for impaired glucose tolerance is also an indicator of increased risk of diabetes and cardiovascular disease. ${ }^{89}$

Helping patients achieve a healthier weight is a wellestablished goal of medical treatment. However, there are few pharmacologic treatments available for weight reduction and the majority of these are administered daily (once to three times per day) ${ }^{10}$ with one recently approved once-weekly agent. ${ }^{11}$ In studies of osteoporosis and other chronic disease states, less-frequent dosing regimens have been associated with better adherence. ${ }^{12-14}$ Improved adherence, in turn, is likely to improve the efficacy of pharmacotherapy and long-term health outcomes, including treatments for obesity. In addition to positive effects on glycemic control, glucagon-like peptide-1 receptor agonists (GLP-1 RAs) have demonstrated efficacy in reducing the risk factors for cardiovascular disease, such as body weight, blood pressure, and cholesterol, in patients who are overweight or obese, with or without T2D. ${ }^{15}$

Efpeglenatide is a long-acting GLP-1 RA currently being developed to improve glycemic control in patients with T2D by subcutaneous once-weekly $(\mathrm{QW})$ administration. It comprises a single amino acid-modified exendin conjugated to a fragment crystallizable region of human immunoglobulin 4 using long-acting peptide/protein technology. ${ }^{16-18}$ This type of conjugation extends the duration of action of efpeglenatide, ${ }^{19}$ while the flexible mini-polyethylene glycol linker minimizes the loss of intrinsic activity. ${ }^{16}$ In vitro, this has been shown to result in faster dissociation of efpeglenatide from the GLP-1 receptor, leading to reduced receptor internalization and desensitization, more cell surface receptor availability, and greater intracellular signaling compared with other GLP-1 RAs following chronic exposure. ${ }^{16}$ Efpeglenatide once weekly or once monthly has been shown to significantly improve glycemic control, reduce body weight, and reduce the risk of cardiovascular events in patients with T2D. ${ }^{20-22}$ The BALANCE 205 study (NCT02075281) examined the efficacy of efpeglenatide in patients with obesity or who were overweight with comorbidities and without T2D. ${ }^{23}$ All doses of efpeglenatide examined (QW or once every 2 weeks (Q2W)) significantly reduced body weight from baseline to week 21 versus placebo $(\mathrm{p}<0.0001$; primary endpoint $) .{ }^{23}$ The objective of these exploratory subanalyses was to explore the efficacy and safety of efpeglenatide in subgroups of patients from the BALANCE 205 study, stratified by pre-diabetes status, body mass index (BMI), and age at baseline.

\section{METHODS}

The BALANCE study (NCT02075281) was a 20-week, randomized, placebo-controlled, double-blind, parallelgroup trial (online supplemental figure S1); full study design has been published previously. ${ }^{23}$ All patients provided written informed consent. Details of the ethics review boards are provided in online supplemental table S1. Patients were required to have a BMI $\geq 30 \mathrm{~kg} / \mathrm{m}^{2}$ or a BMI $\geq 27 \mathrm{~kg} / \mathrm{m}^{2}$ with comorbidities and fasting plasma glucose (FPG) $<126 \mathrm{mg} / \mathrm{dL}$. Key exclusion criteria included BMI $>42 \mathrm{~kg} / \mathrm{m}^{2}$, drug-induced (iatrogenic) obesity, known diabetes, or $\mathrm{HbA}_{1 \mathrm{c}}>6.5 \%(48 \mathrm{mmol} / \mathrm{mol})$.

Eligible patients were randomized $(1: 1: 1: 1: 1)$ to efpeglenatide $4 \mathrm{mg}$ QW, $6 \mathrm{mg}$ QW, $6 \mathrm{mg}$ Q2W, $8 \mathrm{mg}$ Q2W, or placebo. Patients were instructed to reduce calorie intake by approximately $500 \mathrm{kcal}$ each day and encouraged to increase physical activity. The primary efficacy endpoint was change from baseline (day 1 or last value observed prior to randomization if this was not available) in body weight to week 21 (ie, after 20 weeks of treatment) ${ }^{23}$

The first exploratory subgroup analysis of BALANCE investigated the efficacy and safety of efpeglenatide in patients with pre-diabetes (defined as $\mathrm{HbA}_{1 \mathrm{c}}$ between $5.7 \%$ and $6.4 \%$ (which directly converts to $39-46 \mathrm{mmol} /$ $\mathrm{mol}$ ) or FPG $100-125 \mathrm{mg} / \mathrm{dL}$ ) at baseline. The endpoints examined included the proportion of patients reverting to normoglycemia (defined as $\mathrm{HbA}_{1 \mathrm{c}}<5.7 \%$ and FPG $<100 \mathrm{mg} / \mathrm{dL}$ ) at the end of the study, as well as change from baseline in $\mathrm{HbA}_{1 c}$, FPG, body weight, waist circumference, total cholesterol, low-density lipoprotein (LDL), high-density lipoprotein (HDL), and triglycerides. Both safety and efficacy endpoints were analyzed in patients with pre-diabetes from the full analysis set, defined as patients who received the study drug and who had at least one assessment recorded after dosing. All patients in the BALANCE study who received the study drug also had at least one assessment recorded, such that the safety set coincided with the full analysis set.

The second exploratory subgroup analysis included all of the patients in the full analysis set of the BALANCE study. Changes from baseline to week 21 versus placebo in body weight and waist circumference were assessed in patients stratified by BMI and age, either $<$ or $\geq$ the median at baseline (median values: $34.9 \mathrm{~kg} / \mathrm{m}^{2}$ and 44 years, respectively).

Gastrointestinal (GI) treatment-emergent adverse events (TEAEs) were described across patient subgroups. The following safety endpoints, which were assessed in the primary analysis, ${ }^{23}$ were also investigated in the pre-diabetes subgroup analysis: incidence of any TEAE, severe TEAE, and symptomatic hypoglycemia (as reported by patients in study diaries, with an alert value of FPG $\leq 70 \mathrm{mg} / \mathrm{dL}$ ), including severe cases (defined as 
hypoglycemia requiring assistance from another person to administer carbohydrates or glucagon actively, or take other corrective actions).

In these exploratory subanalyses, no adjustments were made for multiplicity. All analyses were considered post-hoc and exploratory. Statistical Analysis System (SAS V.9.3) Proc Means was used to calculate the descriptive statistics and SAS Proc Mixed (V.9.3) was used to calculate the least squares (LS) mean and $95.1 \%$ CI. All $\mathrm{p}$ values are descriptive and further studies should be conducted to confirm these results.

\section{RESULTS}

Patient disposition, demographics, and baseline characteristics for the overall BALANCE study population have been published previously. ${ }^{23}$ In the overall population ( $\mathrm{n}=295$ randomized and received treatment), demographic and baseline characteristics were similar across the treatment groups.

A total of 140 patients met the criteria for pre-diabetes at baseline $\left(\mathrm{HbA}_{1 \mathrm{c}} 5.7 \%-6.4 \%\right.$ or FPG $\left.100-125 \mathrm{mg} / \mathrm{dL}\right)$. Demographic and baseline characteristics were similar across the treatment groups (table 1). The median BMI in the overall study population was $34.9 \mathrm{~kg} / \mathrm{m}^{2} ; 146$ patients had a BMI < the median and 149 patients had a BMI $\geq$ the median. The median age was 44 years; 143 patients were $<$ median age and 152 were $\geq$ median age. The numbers of patients below and above the median BMI and age were comparable between the treatment groups (table 1).

In the population with pre-diabetes, three patients discontinued treatment in the efpeglenatide $4 \mathrm{mg}$ QW group, eight in the efpeglenatide $6 \mathrm{mg}$ QW group, ten in the efpeglenatide $6 \mathrm{mg}$ Q2W group, seven in the efpeglenatide $8 \mathrm{mg}$ Q2W group, and five in the placebo group. The main reason for treatment discontinuation was an adverse event (AE): one in the efpeglenatide $4 \mathrm{mg}$ QW group, five in the efpeglenatide $6 \mathrm{mg}$ QW group, four in the efpeglenatide $6 \mathrm{mg}$ Q2W group, five in the efpeglenatide $8 \mathrm{mg}$ Q2W group, and two in the placebo group.

\section{Efficacy outcomes}

\section{Pre-diabetes subgroup analysis}

Among patients meeting the criteria for pre-diabetes at baseline, the proportion of patients reverting to normoglycemia by study end appeared numerically higher with all doses of efpeglenatide, both QW and Q2W, compared with placebo $(64.3 \%, 46.2 \%, 40.6 \%, 41.7 \%$, and $10.0 \%$ for $4 \mathrm{mg}$ QW, $6 \mathrm{mg}$ QW, $6 \mathrm{mg}$ Q2W, $8 \mathrm{mg}$ Q2W, and placebo, respectively; figure 1), although statistical analysis was not performed. In addition, all efpeglenatide doses examined led to greater reductions from baseline to week 21 compared with placebo in $\mathrm{HbA}_{1 \mathrm{c}}$ (LS mean difference for the overall efpeglenatide group $(\mathrm{n}=110)$ vs placebo $(\mathrm{n}=30):-0.35 \% \quad(95.1 \% \mathrm{CI}-0.43$ to -0.26$)$, nominal $\mathrm{p}<0.0001$; figure 2A) and FPG (LS mean difference for the overall efpeglenatide group $(\mathrm{n}=110)$ vs placebo $(\mathrm{n}=30):-11.19 \mathrm{mg} / \mathrm{dL}(-0.62 \mathrm{mmol} / \mathrm{L})(95.1 \% \mathrm{CI}-16.57$ to -5.81$)$, nominal $\mathrm{p}<0.0001$; figure $2 \mathrm{~B})$. LS mean treatment differences in $\mathrm{HbA}_{1 \mathrm{c}}$ for the individual efpeglenatide groups versus placebo ranged from $-0.30 \%$ $(-3.29 \mathrm{mmol} / \mathrm{mol})$ for the $4 \mathrm{mg}$ QW group to $-0.38 \%$ $(-4.21 \mathrm{mmol} / \mathrm{mol}$ ) for the $6 \mathrm{mg}$ Q2W group (all nominal $\mathrm{p}<0.0001$ vs placebo). For FPG, individual LS mean differences between efpeglenatide and placebo ranged from $-7.72 \mathrm{mg} / \mathrm{dL}(-0.43 \mathrm{mmol} / \mathrm{L}$, nominal $\mathrm{p}=0.0221)$ for the $4 \mathrm{mg}$ QW group to $-14.06 \mathrm{mg} / \mathrm{dL}(-0.78 \mathrm{mmol} / \mathrm{L}$, nominal $\mathrm{p}=0.0001$ ) in the $6 \mathrm{mg} \mathrm{Q} 2 \mathrm{~W}$ group.

Greater reductions in body weight (nominal $\mathrm{p}<0.0001$; figure 2C) were also observed with efpeglenatide compared with placebo, and these reductions were comparable in patients with or without pre-diabetes (figure 2C). In the efpeglenatide $4 \mathrm{mg} \mathrm{QW}, 6 \mathrm{mg} \mathrm{QW}$, $6 \mathrm{mg}$ Q2W, $8 \mathrm{mg}$ Q2W, and placebo groups, the absolute LS mean reductions in weight in those with pre-diabetes were $-5.7 \mathrm{~kg},-7.3 \mathrm{~kg},-6.6 \mathrm{~kg},-7.4 \mathrm{~kg}$, and $-0.1 \mathrm{~kg}$, respectively. The LS mean differences in weight loss between the efpeglenatide groups and the placebo group ranged from $-5.61 \mathrm{~kg}$ in the $4 \mathrm{mg}$ QW group to $-7.32 \mathrm{~kg}$ in the $8 \mathrm{mg}$ Q2W dose group (nominal $\mathrm{p}<0.0001$ for all groups). Efpeglenatide led to greater reductions in waist circumference compared with placebo (LS mean difference vs placebo of $-3.1 \mathrm{~cm},-4.7 \mathrm{~cm},-5.2 \mathrm{~cm}$, and $-7.7 \mathrm{~cm}$ for $4 \mathrm{mg}$ QW, $6 \mathrm{mg}$ QW, $6 \mathrm{mg}$ Q2W, and $8 \mathrm{mg}$ Q2W, respectively; nominal $\mathrm{p}<0.05$ for all except $4 \mathrm{mg} \mathrm{QW}$ ) (online supplemental figure S2).

Compared with placebo, all doses of efpeglenatide led to greater reductions in total cholesterol from baseline to week 21 (LS mean difference vs placebo of $-15.4 \mathrm{mg} / \mathrm{dL}$ (nominal $\mathrm{p}=0.02$ ),$-19.7 \mathrm{mg} / \mathrm{dL}$ (nominal $\mathrm{p}=0.04),-14.9 \mathrm{mg} / \mathrm{dL}$ (nominal $\mathrm{p}=0.02$ ), and $-14.0 \mathrm{mg} /$ $\mathrm{dL}$ (nominal $\mathrm{p}=0.04$ ) for $4 \mathrm{mg} \mathrm{QW}, 6 \mathrm{mg} \mathrm{QW}, 6 \mathrm{mg}$ Q2W, and $8 \mathrm{mg}$ Q2W, respectively) (online supplemental figure S3). With the exception of the effects of the efpeglenatide $6 \mathrm{mg}$ QW dose on LDL (LS mean change vs placebo of $-20.0 \mathrm{mg} / \mathrm{dL}$, nominal $\mathrm{p}=0.002$ ), reductions in LDL, HDL, and triglycerides with efpeglenatide did not achieve nominal statistical significance compared with placebo.

\section{BMI and age subgroup analysis}

Across all BMI and age subgroups (either $<$ or $\geq$ median), all doses of efpeglenatide were associated with greater reductions in body weight (nominal $\mathrm{p}<0.01$; figure $3 \mathrm{~A}$ ) from baseline to week 21 compared with placebo. In patients with age $<$ median, mean weight changes of $-7.8 \mathrm{~kg},-7.6 \mathrm{~kg},-6.6 \mathrm{~kg},-6.8 \mathrm{~kg}$, and $0.5 \mathrm{~kg}$ were observed in the $4 \mathrm{mg} \mathrm{QW}, 6 \mathrm{mg} \mathrm{QW}, 6 \mathrm{mg}$ Q2W, $8 \mathrm{mg}$ Q2W, and placebo groups, respectively. The LS mean difference in body weight between the efpeglenatide groups and placebo in patients with age $<$ median was $-8.39 \mathrm{~kg}$, $-7.55 \mathrm{~kg},-7.27 \mathrm{~kg}$, and $-7.53 \mathrm{~kg}$ (nominal $\mathrm{p}<0.0001$ for all doses) in the $4 \mathrm{mg}$ QW (n=28), $6 \mathrm{mg}$ QW (n=31), $6 \mathrm{mg}$ Q2W (n=28), and $8 \mathrm{mg}$ Q2W (n=28) dose groups, 
Table 1 Demographics and baseline characteristics

\section{Patients meeting the criteria for pre-diabetes}

\begin{tabular}{|c|c|c|c|c|c|}
\hline \multirow[b]{2}{*}{ Characteristics, mean (SD) } & \multicolumn{4}{|l|}{ Efpeglenatide } & \multirow[b]{2}{*}{$\begin{array}{l}\text { Placebo } \\
(n=30)\end{array}$} \\
\hline & $\begin{array}{l}4 \mathrm{mg} \mathrm{QW} \\
(\mathrm{n}=28)\end{array}$ & $\begin{array}{l}6 \mathrm{mg} Q W \\
(\mathrm{n}=26)\end{array}$ & $\begin{array}{l}6 \mathrm{mg} Q 2 W \\
(\mathrm{n}=32)\end{array}$ & $\begin{array}{l}8 \mathrm{mg} Q 2 W \\
(\mathrm{n}=24)\end{array}$ & \\
\hline Female, n (\%) & $18(64.3)$ & $20(76.9)$ & $23(71.9)$ & $22(91.7)$ & $23(76.7)$ \\
\hline Male, n (\%) & $10(35.7)$ & $6(23.1)$ & $9(28.1)$ & $2(8.3)$ & $7(23.3)$ \\
\hline \multicolumn{6}{|l|}{ Race, n (\%) } \\
\hline White & $21(75.0)$ & $13(50.0)$ & $25(78.1)$ & $17(70.8)$ & $17(56.7)$ \\
\hline Black or African American & $5(17.9)$ & $6(23.1)$ & $4(12.5)$ & $3(12.5)$ & $9(30.0)$ \\
\hline Asian & $1(3.6)$ & $6(23.1)$ & $2(6.3)$ & $4(16.7)$ & $3(10.0)$ \\
\hline $\begin{array}{l}\text { Native Hawaiian or other Pacific } \\
\text { Islander }\end{array}$ & $1(3.6)$ & $0(0)$ & $0(0)$ & $0(0)$ & $0(0)$ \\
\hline Other & $0(0)$ & $1(3.8)$ & $1(3.1)$ & $0(0)$ & $1(3.3)$ \\
\hline \multicolumn{6}{|l|}{ Ethnicity, n (\%) } \\
\hline Hispanic or Latino & $3(10.7)$ & $3(11.5)$ & $2(6.3)$ & $2(8.3)$ & $3(10.0)$ \\
\hline Not Hispanic or Latino & 25 (89.3) & $23(88.5)$ & $30(93.8)$ & $22(91.7)$ & $27(90.0)$ \\
\hline Age, years & $45.6(11.0)$ & $46.2(12.2)$ & $48.5(10.9)$ & $47.5(9.3)$ & $45.0(11.3)$ \\
\hline Weight, kg & $104.9(22.0)$ & $104.5(23.3)$ & $100.9(19.5)$ & $94.5(11.3)$ & $96.9(10.9)$ \\
\hline $\mathrm{BMI}, \mathrm{kg} / \mathrm{m}^{2}$ & $35.9(4.7)$ & $36.8(5.0)$ & $35.9(5.6)$ & $34.8(3.3)$ & $35.1(3.1)$ \\
\hline Waist circumference, $\mathrm{cm}$ & $112.0(13.1)$ & $113.4(14.8)$ & $113.0(12.6)$ & $108.7(10.4)$ & $109.7(9.8)$ \\
\hline $\mathrm{HbA}_{1 \mathrm{c}}, \%$ & $5.7(0.3)$ & $5.7(0.3)$ & $5.7(0.3)$ & $5.7(0.3)$ & $5.7(0.4)$ \\
\hline $\mathrm{HbA}_{1 \mathrm{c}}, \mathrm{mmol} / \mathrm{mol}$ & $38.6(3.1)$ & $38.3(3.2)$ & $38.9(2.8)$ & $39.1(3.2)$ & $38.8(4.0)$ \\
\hline $\mathrm{FPG}, \mathrm{mg} / \mathrm{dL}$ & $100.3(9.9)$ & $101.7(9.6)$ & $103.4(10.0)$ & $103.3(8.5)$ & $102.3(12.0)$ \\
\hline Total cholesterol, mg/dL & $185.1(29.3)$ & $183.2(36.2)$ & $193.2(32.2)$ & $192.4(27.8)$ & $180.3(28.3)$ \\
\hline BALANCE 205 full analysis set & $\begin{array}{l}4 \mathrm{mg} \mathrm{QW} \\
(\mathrm{n}=59)\end{array}$ & $\begin{array}{l}6 \mathrm{mg} Q W \\
(\mathrm{n}=59)\end{array}$ & $\begin{array}{l}6 \mathrm{mg} \text { Q2W } \\
(\mathrm{n}=59)\end{array}$ & $\begin{array}{l}8 \mathrm{mg} Q 2 W \\
(\mathrm{n}=58)\end{array}$ & $\begin{array}{l}\text { Placebo } \\
(n=60)\end{array}$ \\
\hline Mean age, years (range) & $42.9(20-64)$ & $43.0(18-64)$ & $43.3(20-64)$ & $43.9(27-64)$ & $43.7(18-64)$ \\
\hline$<$ median, $\mathrm{n}$ & 28 & 31 & 28 & 28 & 28 \\
\hline$\geq$ median, $\mathrm{n}$ & 31 & 28 & 31 & 30 & 32 \\
\hline Female, n (\%) & $41(69.5)$ & $46(78.0)$ & $43(72.9)$ & $51(87.9)$ & 44 (73.3) \\
\hline \multicolumn{6}{|l|}{ Race, n (\%) } \\
\hline White & $41(69.5)$ & $37(62.7)$ & 45 (76.3) & $39(67.2)$ & $39(65.0)$ \\
\hline Black or African American & $12(20.3)$ & $11(18.6)$ & $8(13.6)$ & $13(22.4)$ & $16(26.7)$ \\
\hline Asian & $4(6.8)$ & $8(13.6)$ & $5(8.5)$ & $5(8.6)$ & $3(5.0)$ \\
\hline $\begin{array}{l}\text { American Indian or Alaska } \\
\text { Native }\end{array}$ & $0(0)$ & $0(0)$ & $0(0)$ & $0(0)$ & $1(1.7)$ \\
\hline $\begin{array}{l}\text { Native Hawaiian or other Pacific } \\
\text { Islander }\end{array}$ & $1(1.7)$ & $0(0)$ & $0(0)$ & $1(1.7)$ & $0(0)$ \\
\hline Other & $0(0)$ & $2(3.4)$ & $1(1.7)$ & $0(0)$ & $1(1.7)$ \\
\hline $\begin{array}{l}\text { Multiple: White, Black, or African } \\
\text { American }\end{array}$ & $1(1.7)$ & $1(1.7)$ & $0(0)$ & $0(0)$ & $0(0)$ \\
\hline \multicolumn{6}{|l|}{ Ethnicity, n (\%) } \\
\hline Hispanic or Latino & $4(6.8)$ & $10(16.9)$ & $7(11.9)$ & $6(10.3)$ & $7(11.7)$ \\
\hline Not Hispanic or Latino & 55 (93.2) & 49 (83.1) & $52(88.1)$ & $52(89.7)$ & $53(88.3)$ \\
\hline Mean $\mathrm{HbA}_{1 \mathrm{c}}, \%$ (range) & $5.5(4.6-6.2)$ & $5.4(4.6-6.5)$ & $5.5(4.9-6.1)$ & $5.4(4.5-6.2)$ & $5.5(4.4-6.5)$ \\
\hline Mean $\mathrm{HbA}_{1 \mathrm{c}}$, $\mathrm{mmol} / \mathrm{mol}$ (range) & $36.1(26.8-44.3)$ & $35.3(26.8-47.5)$ & $36.9(30.1-43.2)$ & $35.9(25.7-44.3)$ & $36.4(24.6-47.5)$ \\
\hline Mean weight, kg (range) & $100.8(71.6-191.0)$ & $101.7(64.4-157.3)$ & $99.5(71.4-161.0)$ & $95.6(73.1-138.3)$ & $97.5(67.0-130.0)$ \\
\hline Mean BMI, kg/m² (range) & $35.2(28.3-53.1)$ & $36.3(28.1-49.7)$ & $35.6(28.1-57.7)$ & $35.2(28.6-45.2)$ & $34.9(28.2-41.7)$ \\
\hline$<$ median, $\mathrm{n}$ & 32 & 23 & 28 & 32 & 31 \\
\hline$\geq$ median, $\mathrm{n}$ & 27 & 36 & 31 & 26 & 29 \\
\hline Mean waist circumference (range) & $109.8(87.4-154.5)$ & $111.9(86.0-147.3)$ & $109.6(89.0-141.0)$ & $108.9(85.0-134.5)$ & $109.3(83.0-134.0)$ \\
\hline
\end{tabular}

$\mathrm{BMI}$, body mass index; FPG, fasting plasma glucose; $\mathrm{HbA}_{10}$, glycated hemoglobin; Q2W, once every 2 weeks; QW, once weekly. 


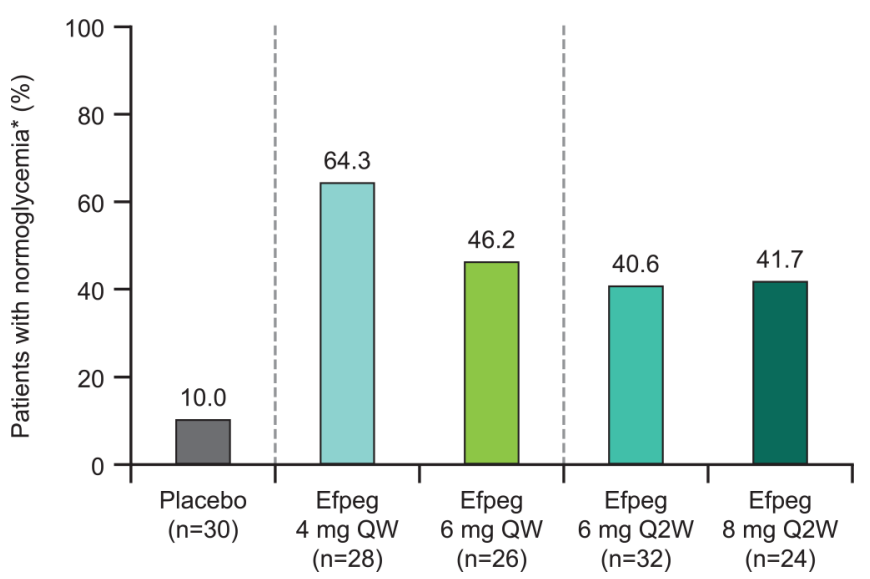

Figure 1 Proportion of patients with pre-diabetes reverting to normoglycemia at the end of the study. *No longer prediabetic, defined as $\mathrm{HbA}_{1 \mathrm{c}}<5.7 \%$ and $\mathrm{FPG}<100 \mathrm{mg} / \mathrm{dL}$ and both not missing at the end of treatment (week 21). Efpeg, efpeglenatide; FPG, fasting plasma glucose; $\mathrm{HbA}_{1 \mathrm{c}}$, glycated hemoglobin; QW, once weekly; Q2W, once every 2 weeks.

respectively. In patients with age $\geq$ median, mean decreases in weight of $-5.8 \mathrm{~kg},-8.2 \mathrm{~kg},-6.8 \mathrm{~kg},-7.4 \mathrm{~kg}$, and $-0.8 \mathrm{~kg}$ were observed in the $4 \mathrm{mg}$ QW, $6 \mathrm{mg}$ QW, $6 \mathrm{mg}$ Q2W, $8 \mathrm{mg}$ Q2W, and placebo groups, respectively.
The LS mean difference in body weight between the efpeglenatide groups and placebo in patients with age $\geq$ median was $-5.21 \mathrm{~kg},-7.18 \mathrm{~kg},-5.56 \mathrm{~kg}$, and $-6.44 \mathrm{~kg}$ (nominal $\mathrm{p}<0.0001 \mathrm{vs}$ placebo for all doses) in the $4 \mathrm{mg}$ QW (n=31), 6mg QW (n=28), 6mg Q2W (n=31), and $8 \mathrm{mg}$ Q2W (n=30) dose groups, respectively. Greater reductions in waist circumference were also observed with efpeglenatide compared with placebo across subgroups (figure 3B; nominal $\mathrm{p}<0.05$ for all except the lower QW and Q2W doses in subgroups with BMI $\geq$ median (4 mg QW and $6 \mathrm{mg}$ Q2W) and age $\geq$ median (4mg QW)).

\section{Safety outcomes}

\section{Pre-diabetes subgroup analysis}

In patients with pre-diabetes at baseline, TEAEs were reported in $85.7 \%, 88.5 \%, 96.9 \%, 87.5 \%$, and $80.0 \%$ of the efpeglenatide $4 \mathrm{mg}$ QW, $6 \mathrm{mg}$ QW, $6 \mathrm{mg}$ Q2W, $8 \mathrm{mg}$ Q2W, and placebo groups, respectively (online supplemental table S2). As expected with treatment with a GLP-1 RA, GI disorders were more common in the efpeglenatide-treated groups compared with placebo; the rates of GI TEAEs were $64.3 \%, 76.9 \%$, $71.9 \%, 70.8 \%$, and $40.0 \%$ in the efpeglenatide $4 \mathrm{mg}$ QW, $6 \mathrm{mg}$ QW, $6 \mathrm{mg}$ Q2W, $8 \mathrm{mg}$ Q2W, and placebo

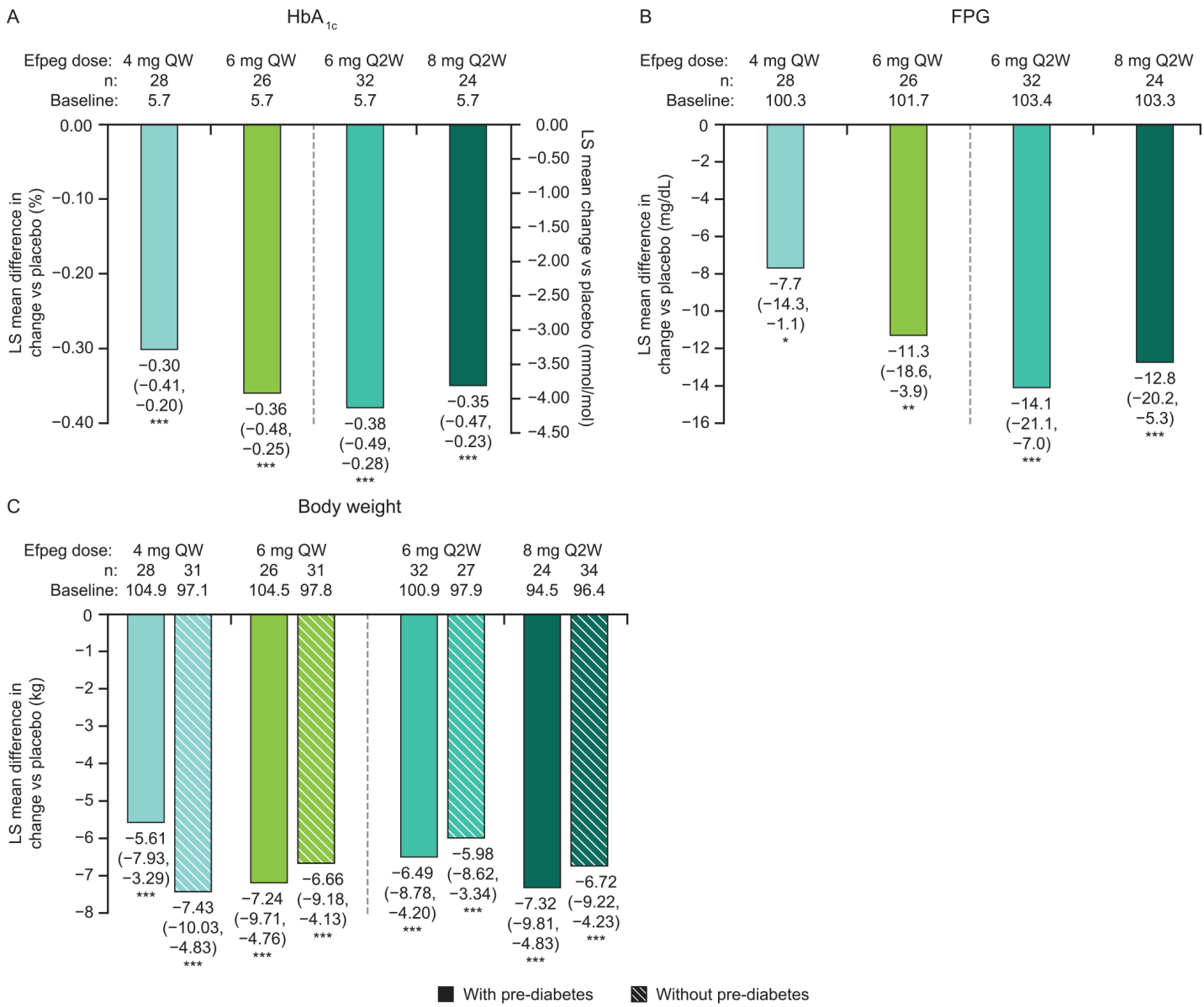

Figure 2 LS mean difference in change from baseline to week 21 versus placebo in (A) $\mathrm{HbA}_{1 \mathrm{c}}$ (patients with pre-diabetes only), (B) FPG (patients with pre-diabetes only), and (C) body weight (patients with and without pre-diabetes). 95.1\% $\mathrm{Cl}$ data are shown in brackets. $(A){ }^{* * *} p<0.0001$. $(B, C){ }^{*} p<0.05,{ }^{* *} p<0.01,{ }^{* * *} p<0.001$. Efpeg, efpeglenatide; FPG, fasting plasma glucose; $\mathrm{HbA}_{1 \mathrm{c}}$, glycated hemoglobin; LS, least squares; QW, once weekly; Q2W, once every 2 weeks. 
A

Body weight $(\mathrm{kg})$

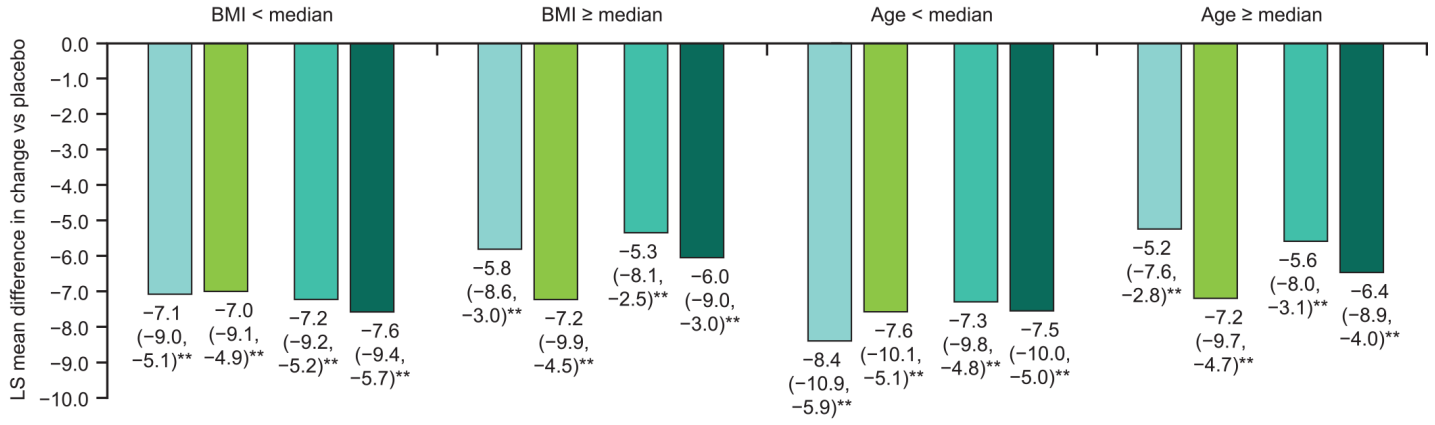

Waist circumference $(\mathrm{cm})$

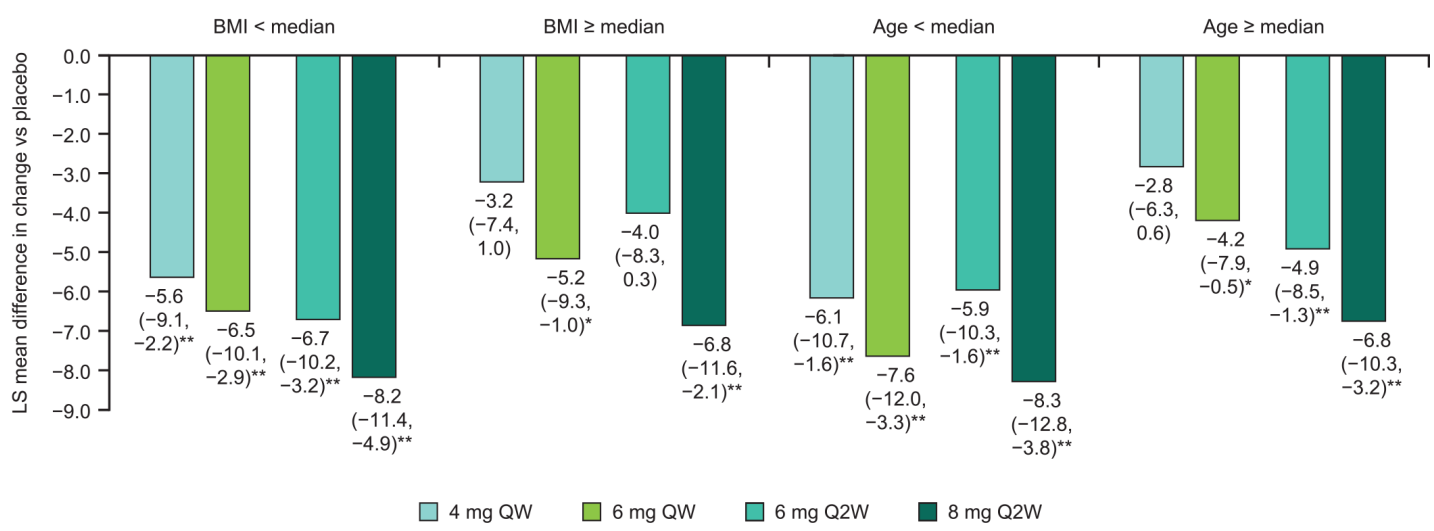

Figure 3 Patients stratified by BMI and age at baseline: LS mean difference in change from baseline to week 21 vs placebo in (A) body weight and (B) waist circumference. $95.1 \% \mathrm{Cl}$ data are shown in brackets. Median $\mathrm{BMI}=34.9 \mathrm{~kg} / \mathrm{m}^{2}$; median age $=44$ years. ${ }^{*} p<0.05$, ${ }^{* *} p<0.01$. BMl, body mass index; LS, least squares; QW, once weekly; Q2W, once every 2 weeks.

groups, respectively. While the incidence of nausea and vomiting was greater with efpeglenatide versus placebo, the incidence of diarrhea was generally greater with placebo versus efpeglenatide groups in patients with pre-diabetes at baseline (online supplemental table S2). The rates of serious TEAEs were generally low and no serious TEAEs were reported in either the placebo or efpeglenatide $6 \mathrm{mg}$ Q2W group. One case of symptomatic hypoglycemia was reported, in the efpeglenatide $6 \mathrm{mg}$ QW group; the event was not severe.

\section{$\mathrm{BMI}$ and age subgroup analysis}

Similar to patients with pre-diabetes at baseline and consistent with the overall BALANCE population, the most common TEAEs in patient subgroups stratified by BMI or age were GI TEAEs such as nausea (incidence range: efpeglenatide, $38.7 \%-73.3 \%$; placebo, $14.3 \%-21.9 \%$ ), vomiting (efpeglenatide, $12.9 \%-40.0 \%$; placebo, $3.6 \%-9.4 \%$ ), and diarrhea (efpeglenatide, $5.6 \%-43.5 \%$; placebo, $17.2 \%-22.6 \%$ ) (online supplemental table S3). No events of pancreatitis were observed. Increases in amylase and lipase were uncommon overall (online supplemental table S3).

\section{DISCUSSION}

Here we have shown data from two subanalyses of the BALANCE study stratified by pre-diabetes status, BMI, and age at baseline. These are commonly observed clinical variables in patients with diabetes and these subanalyses could help to identify response to efpeglenatide in particular patient subgroups.

In patients who had pre-diabetes at baseline, all doses of efpeglenatide led to numerically greater proportions of patients reverting to normoglycemia at the end of the study versus placebo. We observed some potential differences between efpeglenatide groups in terms of achievement of normoglycemia, although statistical testing was not performed. The mean baseline $\mathrm{HbA}_{1 \mathrm{c}}$ and FPG of the group with pre-diabetes were approximately $5.7 \%(39 \mathrm{mmol} / \mathrm{mol})$ and $102 \mathrm{mg} / \mathrm{dL}(5.7 \mathrm{mmol} / \mathrm{L})$, respectively. This is close to the lower limit of prediabetes as defined by the American Diabetes Association $\left(\mathrm{HbA}_{1 \mathrm{c}} 5.7 \%-6.4 \%(39-47 \mathrm{mmol} / \mathrm{mol})\right.$ and $\mathrm{FPG}$ of $100-125 \mathrm{mg} / \mathrm{dL}(5.6-6.9 \mathrm{mmol} / \mathrm{L})),{ }^{8}$ suggesting that some patients in the pre-diabetes group were very close to being normoglycemic. This may explain, at least in part, the high proportions of patients returning to normoglycemia following efpeglenatide treatment and why the highest rate of reversion was observed with the $4 \mathrm{mg}$ QW dose, despite larger reductions in $\mathrm{HbA}_{1 \mathrm{c}}$ and FPG in the other efpeglenatide dose groups.

Treatment with efpeglenatide was also associated with greater improvements from baseline in $\mathrm{HbA}_{1 \mathrm{c}}$, FPG, body weight, waist circumference, and total cholesterol compared with placebo. Due to the exploratory nature 
of the study design, statistical testing was not performed to compare efpeglenatide groups.

In subgroups of patients from the overall BALANCE population, stratified by baseline BMI or age, all doses of efpeglenatide were associated with greater reductions in body weight versus placebo across all subgroups of patients stratified by age and BMI. For waist circumference, the highest $\mathrm{QW}$ and Q2W doses of efpeglenatide were associated with reductions versus placebo. Therefore, these exploratory data suggest that efpeglenatide may be beneficial in people with pre-diabetes and that this is an area that requires further study. The results of these subgroup analyses were consistent with those of the overall BALANCE study, with all doses of efpeglenatide associated with greater reductions in body weight compared with placebo across all patient subgroups examined. ${ }^{23}$ Similarly, the benefits of efpeglenatide on glycemic control and cholesterol levels seen in BALANCE ${ }^{23}$ were also seen in patients with pre-diabetes, who experienced greater reductions in $\mathrm{HbA}_{1 \mathrm{c}}$, FPG, and total cholesterol, compared with patients receiving placebo. In the overall BALANCE study population, all doses of efpeglenatide led to greater reductions in waist circumference compared with placebo; ${ }^{23}$ this treatment effect was seen with most doses of efpeglenatide across the subgroups investigated, with more consistent benefits observed at the higher doses examined. In line with the overall BALANCE population and the GLP-1 RA class, the most common AEs in these subgroup analyses were GI AEs such as nausea, vomiting, and diarrhea. The goal of treatment for pre-diabetes is preventing progression to diabetes, and lifestyle modification is considered as the first-line therapy. ${ }^{245}$ Intensive behavioral lifestyle intervention programs have been shown to reduce the risk of progression to diabetes. ${ }^{25}$ Several pharmacologic agents, primarily metformin, have also been shown to reduce the risk of disease progression, although further study is needed. In practice, however, pre-diabetes often goes unaddressed in primary care settings. ${ }^{26}$

Currently, there are two GLP-1 RAs approved for body weight management in patients with obesity and without diabetes: liraglutide, administered as a oncedaily injection; ${ }^{27}$ and semaglutide, administered QW. ${ }^{11}$ In a trial in patients with obesity and without T2D, 20-week treatment with liraglutide $1.8-3.0 \mathrm{mg}$ once daily led to an $84 \%-96 \%$ decrease in the prevalence of pre-diabetes (defined as FPG 5.6-6.9 mmol/L (100$125 \mathrm{mg} / \mathrm{dL})$ or glucose tolerance $7.8-11.0 \mathrm{mmol} / \mathrm{L}$ (140-199 mg/dL) measured during an oral glucose tolerance test). ${ }^{28}$ Liraglutide was also shown to reduce the incidence of progression to T2D in a study of adults with pre-diabetes and a BMI $\geq 30 \mathrm{~kg} / \mathrm{m}^{2}$ (or $\geq 27 \mathrm{~kg} / \mathrm{m}^{2}$ with comorbidities). Of patients treated with liraglutide, $2 \%$ were diagnosed with diabetes after 3 years compared with $6 \%$ of the placebo group, and the time from randomization to diagnosis was 2.7 times longer with liraglutide than with placebo $(p<0.0001){ }^{29}$ The recent Semaglutide Treatment Effect in People with Obesity (STEP) phase III clinical trial program explored the efficacy and safety of once-weekly semaglutide in overweight adults without diabetes with a BMI of $\geq 30 \mathrm{~kg} / \mathrm{m}^{2}$ or $\geq 27 \mathrm{~kg} / \mathrm{m}^{2}$ (STEP 1 , STEP 3 , and STEP 4$)^{30-32}$ or in adults with T2D with BMI $\geq 27 \mathrm{~kg} / \mathrm{m}^{2}$ (STEP 2) ${ }^{33}$ All trials showed that semaglutide was associated with significantly greater body weight reductions and a greater proportion of patients achieved weight loss of $5 \%$ or greater compared with placebo. ${ }^{30-33}$ The STEP 1 study also showed that, of patients with prediabetes, $84.1 \%$ of those in the once-weekly semaglutide group reverted to normoglycemia after 68 weeks of treatment, compared with $47.8 \%$ in the placebo group. ${ }^{30}$

In the BALANCE study, the rates of GI AEs (ie, nausea, vomiting, diarrhea, dyspepsia, and constipation) with efpeglenatide in the pre-diabetes population were consistent with those in the overall study population ${ }^{23}$; these rates were similar to those observed with liraglutide and semaglutide in phase II studies in patients with obesity. ${ }^{2834}$ In the present analysis, the rates of GI AEs were generally slightly higher in patients with BMI below the median compared with BMI at the median or greater. Although one study found lower weight to be associated with GI AEs in univariate analyses among patients taking liraglutide, another found similar rates of GI AEs in patients with BMI $<35 \mathrm{~kg} / \mathrm{m}^{2}$ compared with BMI $\geq 35 \mathrm{~kg} / \mathrm{m}^{2}$. 35.36 Statistical comparisons of GI AEs between the different efpeglenatide dose groups were not performed in either subgroup analysis.

The limitations of the BALANCE 205 study have been described previously. ${ }^{23}$ This was a phase II study with associated limitations in terms of sample size and study duration. The small sample size could have limited the statistical power needed to detect significance for some endpoints; this issue of small sample size was exacerbated further by dividing the sample into even smaller subgroups. Small group sizes and large variability may have contributed to the finding that reductions in total cholesterol were significantly greater with all doses of efpeglenatide versus placebo, but the differences in change in LDL, HDL, and triglycerides were not. Finally, adjustments for multiple comparisons were not used for these exploratory analyses. As such, the $\mathrm{p}$ values reported are nominal and these results are considered hypothesisgenerating. Further research is needed to confirm these outcomes.

Overall, the findings from these subgroup analyses are consistent with those of the BALANCE study and suggest that efpeglenatide leads to improvements in weight reduction in key subgroups of patients with obesity or who are overweight (with comorbidity) and without diabetes. They suggest that treatment effects are independent of baseline characteristics such as patient age or BMI. In addition, the benefits of efpeglenatide on glycemic control in patients with pre-diabetes suggest 
that it may help patients at risk of developing diabetes to achieve normoglycemia. These promising results would need to be replicated in larger, long-term studies in patients with pre-diabetes. However, while these findings are hypothesis-generating, they support further investigation and development of efpeglenatide in patients with obesity and who are overweight (with comorbidity) and without diabetes, and suggest a potential role of efpeglenatide in the management of pre-diabetes and obesity.

\section{Author affiliations}

${ }^{1}$ Translational Research Institute, AdventHealth Central Florida, Orlando, Florida, USA

${ }^{2}$ Cardio-Metabolic-Institute, Praxis für Prävention und Therapie, VillingenSchwenningen, Germany

${ }^{3}$ Hanmi Pharmaceutical, Seoul, Republic of Korea

${ }^{4}$ ProSciento, Chula Vista, California, USA

${ }^{5}$ Sanofi Canada, Laval, Quebec, Canada

${ }^{6}$ Sanofi, Bridgewater, New Jersey, USA

${ }^{7}$ Seoul St Mary's Hospital, The Catholic University of Korea, Seoul, Republic of Korea

Presented at

Pratley RE, Jacob S, Kang J, Trautmann ME, Hompesch M, Han 0, et al. Efficacy of efpeglenatide in patients with obesity and prediabetes: a subanalysis of the BALANCE 205 study (ADA Abstract). Diabetes. 2019;68(Suppl 1):53-0R. Available from: https://diabetes.diabetesjournals.org/content/68/Supplement_1/53OR. Dailey III GE, Rosenstock J, Morales Sr. C, Wendisch U, Trautmann ME, Hompesch M, et al. Do baseline characteristics impact efficacy of efpeglenatide QW in uncontrolled type 2 diabetes? (ADA Abstract). Diabetes. 2019;68(Suppl 1):1023-P. Available from: https://diabetes.diabetesjournals.org/content/68/ Supplement_1/1023-P. Ogbaa I, Pratley RE, Jacob S, Kang J, Trautmann ME, Hompesch M, et al. Efficacy of efpeglenatide in patients with obesity and prediabetes: a subanalysis of the BALANCE 205 study (EASD Abstract). 2019:ePoster 765. Available from: https://www.easd.org/virtualmeeting/home. html\#!resources/efficacy-of-efpeglenatide-in-patients-with-obesity-andprediabetes-a-subanalysis-of-the-balance-205-study-77797446-1167-4f29-ab5870bc10931855. Sorli CH, Pratley RE, Jacob S, Kang J, Trautmann ME, Hompesch $\mathrm{M}$, et al. Effects of efpeglenatide on weight, BMI, and waist circumference in those with obesity without diabetes stratified by baseline characteristics: subanalysis of BALANCE 205 (EASD Abstract). 2019:ePoster 763. Available from: https://www. easd.org/virtualmeeting/home.html\#!resources/effects-of-efpeglenatide-onweight-bmi-and-waist-circumference-in-those-with-obesity-without-diabetesstratified-by-baseline-characteristics-subanalysis-of-balance- 205.

Acknowledgements In November 2015 Sanofi obtained an exclusive license from Hanmi Pharmaceutical for the worldwide development and commercialization of efpeglenatide, an experimental, long-acting diabetes treatment. The BALANCE 205 trial was conducted by Hanmi Pharmaceutical between April 2014 and February 2015. Editorial assistance was provided by Jennifer Steeber, PhD, of Caudex (New York, New York, USA), and Margarita Lens, MSci, of Fishawack Communications, a Fishawack Health Company.

Contributors REP and SJ contributed to data acquisition, data analysis/ interpretation, and critical revision of the manuscript for important intellectual content. SB, MET, MH, OH, JS, CHS, AS, and K-HY contributed to data analysis/ interpretation and critical revision of the manuscript for important intellectual content. REP is the guarantor and accepts full responsibility for the work and/or the conduct of the study, had access to the data, and controlled the decision to publish.

Funding The study was funded by Hanmi Pharmaceuticals and Sanofi. Editorial assistance was funded by Sanofi.

Competing interests REP has served as a consultant for AstraZeneca, Glytec, Janssen Pharmaceuticals, Merck, MundiPharma, Novo Nordisk, Pfizer, Sanofi, Sanofi US Services, Scohia Pharma, and Sun Pharmaceutical Industries; has received grants and research support from Hanmi Pharmaceutical, Janssen Pharmaceuticals, Metavention, Novo Nordisk, Poxel SA, and Sanofi; and has received honoraria from Novo Nordisk; honoraria and fees for these activities were directed to a non-profit organization with the exception of those from Sanofi US Services, which were personal fees. SB is an employee of Hanmi Pharmaceutical.
MET is a consultant of ProSciento; has received consulting fees from Atrogi, CeQur SA, Hanmi Pharmaceutical, Kinexum, Novo Nordisk, and Servier; and is on the Data Monitoring Board for Profil. MH is an employee and shareholder of ProSciento and has received grants from Hanmi Pharmaceutical. $\mathrm{OH}$ is an employee of Hanmi Pharmaceutical. JS is an employee and shareholder of Sanofi. AS was an employee of Sanofi during the development of this publication. CHS was an employee and shareholder of Sanofi during the development of this publication. SJ has received personal fees from AstraZeneca, Boehringer Ingelheim, Lilly, MSD, Novo Nordisk, and Sanofi. K-HY has nothing to declare.

Patient consent for publication Not required.

Ethics approval The BALANCE study was designed and conducted in accordance with the International Conference on Harmonisation Harmonised Tripartite Guideline for Good Clinical Practice and the Declaration of Helsinki. The protocol was approved by the institutional review boards or ethics committee at each study site.

Provenance and peer review Not commissioned; externally peer reviewed.

Data availability statement Data are available upon reasonable request. The datasets generated during and/or analysed during the current study are available from the corresponding author on reasonable request.

Supplemental material This content has been supplied by the author(s). It has not been vetted by BMJ Publishing Group Limited (BMJ) and may not have been peer-reviewed. Any opinions or recommendations discussed are solely those of the author(s) and are not endorsed by BMJ. BMJ disclaims all liability and responsibility arising from any reliance placed on the content. Where the content includes any translated material, BMJ does not warrant the accuracy and reliability of the translations (including but not limited to local regulations, clinical guidelines, terminology, drug names and drug dosages), and is not responsible for any error and/or omissions arising from translation and adaptation or otherwise.

Open access This is an open access article distributed in accordance with the Creative Commons Attribution Non Commercial (CC BY-NC 4.0) license, which permits others to distribute, remix, adapt, build upon this work non-commercially, and license their derivative works on different terms, provided the original work is properly cited, appropriate credit is given, any changes made indicated, and the use is non-commercial. See: http://creativecommons.org/licenses/by-nc/4.0/.

\section{ORCID iDs}

Richard E Pratley http://orcid.org/0000-0002-2912-1389

Marcus Hompesch http://orcid.org/0000-0001-5867-2131

\section{REFERENCES}

1 Hruby A, Manson JE, Qi L, et al. Determinants and consequences of obesity. Am J Public Health 2016;106:1656-62.

2 Abarca-Gómez L, Abdeen ZA, Hamid ZA, et al. Worldwide trends in body-mass index, underweight, overweight, and obesity from 1975 to 2016: a pooled analysis of 2416 population-based measurement studies in 128.9 million children, adolescents, and adults. Lancet 2017;390:2627-42

3 Engin A. The definition and prevalence of obesity and metabolic syndrome. Adv Exp Med Biol 2017;960:1-17.

4 Frasca D, Blomberg BB, Paganelli R. Aging, obesity, and inflammatory age-related diseases. Front Immunol 2017;8:8.

5 Jura M, Kozak LP. Obesity and related consequences to ageing. Age 2016;38:23

6 Kushner JA. The role of aging upon $\beta$ cell turnover. J Clin Invest 2013;123:990-5.

7 DeFronzo RA, Eldor R, Abdul-Ghani M. Pathophysiologic approach to therapy in patients with newly diagnosed type 2 diabetes. Diabetes Care 2013;36:S127-38.

8 American Diabetes Association. Chapter 2. classification and diagnosis of diabetes: standards of medical care in diabetes-2021. Diabetes Care 2021;44:S15-33.

9 Zand A, Ibrahim K, Patham B. Prediabetes: why should we care? Methodist Debakey Cardiovasc J 2018;14:289-97.

10 Gadde KM, Apolzan JW, Berthoud H-R. Pharmacotherapy for patients with obesity. Clin Chem 2018;64:118-29.

11 Novo Nordisk. Wegovy semaglutide injection - prescribing information. Available: https://www.novo-pi.com/wegovy.pdf [Accessed 12 Jul 2021].

12 Reginster JY, Rabenda V, Neuprez A. Adherence, patient preference and dosing frequency: understanding the relationship. Bone 2006;38:S2-6.

13 Iglay K, Cao X, Mavros P, et al. Systematic literature review and meta-analysis of medication adherence with once-weekly versus once-daily therapy. Clin Ther 2015;37:1813-21. 
14 Saini SD, Schoenfeld P, Kaulback K, et al. Effect of medication dosing frequency on adherence in chronic diseases. Am J Manag Care 2009;15:e22-33

15 Vilsbøll T, Christensen M, Junker AE, et al. Effects of glucagonlike peptide-1 receptor agonists on weight loss: systematic review and meta-analyses of randomised controlled trials. BMJ 2012;344:d7771

16 Choi IY, Moon MJ, Trautmann ME, et al. In vitro studies to evaluate the receptor kinetics of efpeglenatide vs. other glucagon-like peptide-1 receptor (GLP-1 R) agonists - poster 1090-P, American diabetes association 78th scientific session, 22-26 June, 2018.

$17 \mathrm{Ha} \mathrm{J-H,} \mathrm{Kim} \mathrm{J-E,} \mathrm{Kim} \mathrm{Y-S.} \mathrm{Immunoglobulin} \mathrm{Fc} \mathrm{heterodimer} \mathrm{platform}$ technology: from design to applications in therapeutic antibodies and proteins. Front Immunol 2016;7:394.

18 Hanmi Pharmaceutical Co. Ltd. Long acting protein / peptide discovery platform technology. Available: http://www.hanmipharm com/ehanmi/handler/Rnd-ProjectLapscovery [Accessed 11 Mar 2019].

19 Yoon Kun-Ho, Kang J, Kwon SC, et al. Pharmacokinetic and dosefinding studies on efpeglenatide in patients with type 2 diabetes. Diabetes Obes Metab 2020;22:1292-301.

20 Rosenstock J, Sorli CH, Trautmann ME, et al. Once-weekly efpeglenatide dose-range effects on glycemic control and body weight in patients with type 2 diabetes on metformin or drug naive, referenced to liraglutide. Diabetes Care 2019;42:1733-41.

21 Del Prato S, Kang J, Trautmann ME, et al. Efficacy and safety of once-monthly efpeglenatide in patients with type 2 diabetes: results of a phase 2 placebo-controlled, 16-week randomized dose-finding study. Diabetes Obes Metab 2020;22:1176-86.

22 Gerstein HC, Sattar N, Rosenstock J, et al. Cardiovascular and renal outcomes with efpeglenatide in type 2 diabetes. $N$ Engl J Med 2021;385:896-907.

23 Pratley RE, Kang J, Trautmann ME, et al. Body weight management and safety with efpeglenatide in adults without diabetes: a phase II randomized study. Diabetes Obes Metab 2019;21:2429-39.

24 Tabák AG, Herder C, Rathmann W, et al. Prediabetes: a high-risk state for diabetes development. Lancet 2012;379:2279-90.

25 American Diabetes Association. Chapter 3. prevention or delay of type 2 diabetes: standards of medical care in diabetes-2021. Diabetes Care 2021:44:S34-9.
26 Mainous AG, Tanner RJ, Baker R. Prediabetes diagnosis and treatment in primary care. J Am Board Fam Med 2016;29:283-5.

27 Novo Nordisk. Saxenda - prescribing information. Available: https:// www.novo-pi.com/saxenda.pdf [Accessed 9 Nov 2018]

28 Astrup A, Rössner S, Van Gaal L, et al. Effects of liraglutide in the treatment of obesity: a randomised, double-blind, placebocontrolled study. Lancet 2009;374:1606-16.

29 le Roux CW, Astrup A, Fujioka K, et al. 3 years of liraglutide versus placebo for type 2 diabetes risk reduction and weight management in individuals with prediabetes: a randomised, double-blind trial. Lancet 2017;389:1399-409.

30 Wilding JPH, Batterham RL, Calanna S, et al. Once-weekly semaglutide in adults with overweight or obesity. $N$ Engl $\mathrm{J} \mathrm{Med}$ 2021;384:989-1002.

31 Wadden TA, Bailey TS, Billings LK, et al. Effect of subcutaneous semaglutide vs placebo as an adjunct to intensive behavioral therapy on body weight in adults with overweight or obesity. JAMA 2021;325:1403-13.

32 Rubino D, Abrahamsson N, Davies M, et al. Effect of continued weekly subcutaneous semaglutide vs placebo on weight loss maintenance in adults with overweight or obesity. JAMA 2021;325:1414-25.

33 Davies M, Færch L, Jeppesen OK, et al. Semaglutide 2.4 mg once a week in adults with overweight or obesity, and type 2 diabetes (STEP 2): a randomised, double-blind, double-dummy, placebocontrolled, phase 3 trial. Lancet 2021;397:971-84.

34 O'Neil PM, Birkenfeld AL, McGowan B, et al. Efficacy and safety of semaglutide compared with liraglutide and placebo for weight loss in patients with obesity: a randomised, double-blind, placebo and active controlled, dose-ranging, phase 2 trial. Lancet 2018;392:637-49.

35 Thong KY, Gupta PS, Blann AD, et al. The influence of age and metformin treatment status on reported gastrointestinal side effects with liraglutide treatment in type 2 diabetes. Diabetes Res Clin Pract 2015;109:124-9.

36 le Roux C, Aroda V, Hemmingsson J, et al. Comparison of efficacy and safety of liraglutide $3.0 \mathrm{mg}$ in individuals with $\mathrm{BMI}$ above and below 35 kg/m²: A post-hoc analysis. Obes Facts 2017;10:531-44. 